\title{
A Novel MPPT Algorithm Based on Particle Swarm Optimisation for Photovoltaic Systems
}

\author{
Ramdan B. A. Koad, Ahmed F. Zobaa, Senior Member, IEEE and Adel El-Shahat
}

\begin{abstract}
This paper describes a new maximum-power-pointtracking method for a photovoltaic system based on the Lagrange Interpolation Formula and proposes the particle swarm optimization method. The proposed control scheme eliminates the problems of conventional methods by using only a simple numerical calculation to initialize the particles around the global maximum power point. Hence, the suggested scheme will utilize fewer iterations to reach the maximum power point. The proposed algorithm is verified with the OPAL-RT real time simulator and the Matlab Simulink tool, with several simulations being carried out, and compared to the Perturb and Observe method, the Incremental Conductance method, and the conventional Particle Swarm Optimization based algorithm. The simulation results indicate the proposed algorithm can effectively enhance stability and fast tracking capability under fastchanging non-uniform insolation conditions.
\end{abstract}

Index Terms-Photovoltaic (PV) systems, maximum power point tracking (MPPT), Perturb and Observe (P\&O) Method, Incremental Conductance (IncCond), OPAL-RT, particle swarm optimization (PSO), partial shading conditions (PSC).

\section{INTRODUCTION}

$\mathrm{T}^{\mathrm{s}}$ he power-voltage $(\mathrm{P}-\mathrm{V})$ characteristic of a photovoltaic (PV) module dictates its optimum operating point, the point at which it can deliver maximum power; this is known as the maximum power point (MPP). This point is not constant, but dependent on weather conditions and load impedance. Therefore, maximum-power-point tracking (MPPT) methods are required for a PV system to maintain efficient operation of the PV panels present, at their MPP [1], [2]. Recently, a number of authors offered different explanations for the problems associated with the MPPT controller. Several MPPT methods have been developed thus far, ranging from the simple to the more complex, and dependent on the weather conditions and the control strategies used. Among these are the Perturb and Observe ( $\mathrm{F} \& \mathrm{O})$ method [1] and [3] and the Incremental Conductance (IncCond) method [4], [5]. These algorithms have the advantage of working independently, as knowledge of PV generator characteristics is not critical. Although such methods are simple to implement [7], they are unable to track the MPP accurately in circumstances where levels of solar radiation are changing rapidly. Furthermore, they cannot

The authors are with The College of Engineering, Design and Physical Sciences, Brunel University London, Uxbridge, Middlesex, UB8 3PH, U.K. (e-mail: Ramdan.Koad@Brunel.ac.uk ; azobaa@ieee.org; aahmed@georgiasouthern.edu). operate the system at the MPP under partial shading conditions (PSC), because they lack differentiation between the local MPP and its global peak (GP) [8]- [10].

Reference [11] describes a PV system under PSC, illustrating that the use of a conventional MPPT algorithm under partial shadowing conditions could result in significant power losses. According to [12], the efficiency of MPPT controllers is reduced under PSC, because most MPPT controllers operate such that there is only one point at which the $\mathrm{PV}$ module can produce maximum power within the range of its $\mathrm{P}-\mathrm{V}$ characteristic. However, when PSC occurs, the P-V characteristic becomes more complex, exhibiting multiple peaks, which in turn affect the performance of the controller, reducing the entire output power of the system as a result [6], [7]. Recently, numerous modified MPPT methods have been proposed in the literature to ensure the accurate tracking of MPP, to improve dynamic system response and minimize the system hardware [14], [16]. These methods differ in their complexity, accuracy, and speed. Even if tracking were done perfectly using these methods, the dynamic response speed of the system would still be low [2], [6], [11]. An alternative optimization technique applied to the MPPT controller of a PV system, operating under PSC, is the Particle Swarm Optimization (PSO) algorithm [9], [15]-[18].

The PSO technique exhibits considerable potential, due to its easy implementation, fast computation capability, and its ability to determine the MPP irrespective of environmental conditions. It can also perform a search that is more random than searches performed as part of other evolutionary techniques, such as the Genetic Algorithm (GA). The difference between the PSO algorithm and conventional techniques is that with the PSO method, the updating of the duty cycle based on the particle velocity is not fixed, while when employing other techniques the duty cycle is perturbed by a fixed value. The result is that oscillations occur around the MPP in a steady state, as reported in [9] and [15]-[17]. In standard PSO, particles are usually initialized randomly following uniform distribution over the search space. This requires large time delays to enable the particles to converge towards the MPP, thereby resulting in long computation times [6], [11]. However, a proper initialization of the particles can improve PSO efficiency, resulting in the detection of superior solutions with faster convergence.

As the initialization of the swarm in PSO is a crucial issue affecting performance, the authors of [14] proposed a twostage algorithm. First, they applied the P\&O method to identify the nearest local maximum, and then used the PSO method in the second stage to reach the GP. However, the $\mathrm{P} \& \mathrm{O}$ technique requires longer to determine the MPP. 
Moreover, the $\mathrm{P} \& \mathrm{O}$ technique can become confused under exposure to rapidly changing weather conditions.

In [9], the random numbers of the standard PSO acceleration factors were removed to reduce the search time. However, the change in particle velocity needed to be restricted, as while a low velocity value would impose a need for more iterations to reach the GP, with a large value it may escape the GP.

A re-initialised PSO-IncCond process is suggested in [18]. The IncCond process is employed to discover the locality of MPP. After this, the averages of the function cycle and the output power within the IncCond technique are employed to re-initialise the standards for identifying the finest duty cycles and the highest power rate in the PSO process in that order. Despite the benefits of precise tracking that are possible when using PSO-founded techniques, tracking takes a lot longer than when using traditional processes, particularly under PSC, which is a key drawback.

Ref [19] projected a novel MPPT, founded on the PSO algorithm by adding extra coefficients to model PSO equations to enhance the algorithm computational load. Nevertheless, it is not apparent whether the algorithm will track the right MPP continually, because within the PSO algorithm, as the particles reach the MPP, their speed falls to extremely low or nil. One of the frequently encountered difficulties with the PSO algorithm is that underneath conditions of slow difference in solar emissions, the alteration of the duty cycle needs to be small to track the MPP accurately. Nevertheless, this leads to a definite amount of power needing to be utilized during the investigative process, and determines that the conversion towards the MPP will be gradual. In contrast, if the adjustment to the duty cycle is large, it is then not possible to trace the novel MPP accurately.

In view of these drawbacks, this paper offers a novel approach to augment the MPPT method for the PV system, based on the Lagrange Interpolation (LI) formula and the PSO method. Initially, the LI method is used to determine the optimum value of the duty cycle in the case of the MPP according to the operating point. Starting from that point, the PSO method will then be used to search for the true GP. The proposed MPPT controller essentially initializes the particles around the MPP, thereby providing the initial swarm with information concerning the best position. This can thereby improve PSO efficiency and lead to faster convergence, with zero steady-state oscillations. Additionally, there is no need to restrict particle velocity, because the initial values are closer to the MPP. Thus, the proposed technique aims to increase efficiency without adding any extra complexity, thereby substantially enhancing possible tracking speeds, while also reducing the steady-state oscillation (practically to zero) once the MPP is located. This offers considerable improvements over the conventional PSO method, in which new operating points are too far from the MPP requiring additional iterations.

\section{Terminal Characteristics of Photovoltaic Cells}

The equivalent circuit of the PV module is shown in Fig. 1.

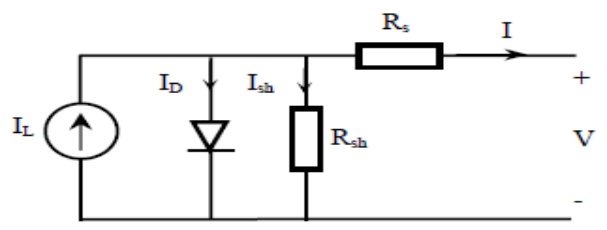

Fig. 1. Single-diode PV cell model with $\boldsymbol{R}_{\boldsymbol{S}}$ and $\mathbf{s h}$ [16].

The corresponding current-voltage (I-V) characteristic equation can be written as follows:

$I=I_{p h}-I_{o}\left\{\left[\exp \left(\frac{\mathrm{q}\left(\mathrm{V}+\mathrm{I} R_{S}\right.}{A K T}\right)-1\right\}-\frac{\mathrm{V}+\mathrm{I} R_{S}}{R_{S h}}\right.$

For the study, the selected PV module is the BP Solar SX 150S PV module, and the proposed system uses the Cùk converter. Equation (2) gives the relationship between the output and input voltages and the duty cycle of the Cùk converter:

$\frac{V_{l o a d}}{V_{p v}}=\frac{D}{1-\mathrm{D}}$

\section{EFFeCt OF THE PARTial Shading CONDitions}

The solar cells in the practical system have been connected in series or parallel configurations to form modules/arrays and generate the desired voltage values. However, the PV module output voltage is determined by the output current generated. This depends chiefly on the solar radiation conditions, as these are directly proportional to irradiance. Therefore, in an application, where there are multiple PV modules working under different irradiance conditions, there will be an opportunity to implement different maximum output power points, instead of a single MPP. This may result in a substantial reduction in output power for the entire system, as the controller might not find the true operating point for the MPP. This condition can occur where there is a partial shading condition [16]-[19].

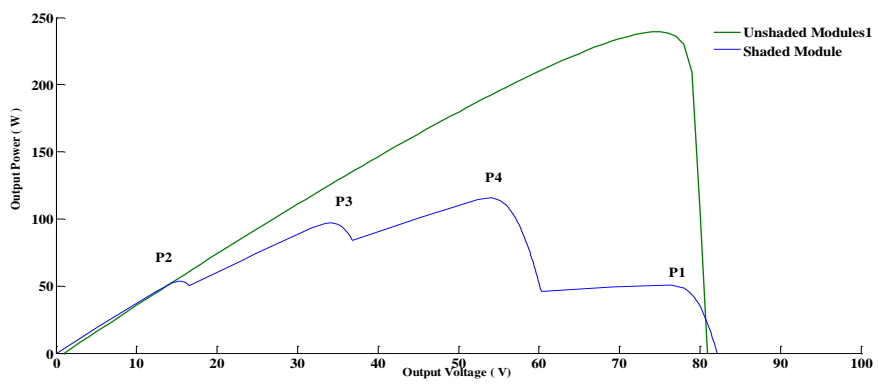

Fig. 2. $V$-P curve of the PV array under PSC.

The simulated PV module is the MSX60, connected in the series-parallel $(4 \times 1)$ configuration. The resulting $P-V$ curve is shown in Fig. 2, when some of the modules in the PV array are shaded. It can be observed that the $P-V$ curve on the PV array exhibits multiple MPPs under this condition.

\section{OVER VIEW OF THE PARTICLE SWARM OPTIMIZATION ALGORITHM}

The PSO algorithm is an optimization technique that can be applied using multivariable function optimization with many local optimal points, as presented by Kennedy and Eberhart in 
1995 [9] and [14]. The principle of the PSO algorithm was inspired by observations of natural social behaviour, such as bird flocking and fish schooling. The key differences between the PSO and other global optimization approaches were the easy implementation and fast convergence of the former. As a result, PSO has received growing attention from researchers studying its use with MPPT in PV systems.

Following the aforementioned flocking analogy, PSO modelled several cooperative "birds," termed particles in this case, acting together in a "flock," otherwise known as a swarm. Each particle in the swarm has a fitness value mapped by an objective function and an individual velocity, which the particle uses to determine the direction and distance of the movement. Each particle exchanges the information obtained through its respective search processes [10], [13] and [14]. The position of a particle is influenced by two variables: the best solution found by the particle itself $\left(p_{\text {best }}\right)$, which is stored for use as individual best position, and the best particle in the neighbourhood $\left(g_{\text {best }}\right)$, which is stored as the best position for the swarm. The particle swarm uses this method to move towards the best position, continuously revising its direction and velocity as needed; following this approach, each particle ultimately moves toward an optimal point or close to a global optimum [14]. The standard PSO method can be defined according to the following equations:

$$
\begin{aligned}
& v_{i}(k+1)=w v_{i}(k)+c_{1} r_{1} \cdot\left(P_{\text {best }}-x_{i}(k)\right)+c_{2} r_{2} \cdot\left(g_{\text {best }}-x_{i}(k)\right) \\
& x_{i}(k+1)=x_{i}(k)+v_{i}(k+1) \\
& i=1,2, \ldots, \mathrm{N}
\end{aligned}
$$

Where $x_{i}$ and $v_{i}$ are the velocity and position of particle $i$, respectively, $k$ represents the iteration number, $w$ is the inertia weight, $r 1$ and $r 2$ are random variables whose values are uniformly distributed in the range [0, 1], and $c 1$ and $c 2$ represent the cognitive and social coefficients, respectively. $p_{\text {best }, i}$ is the individual best position of particle $i$, and $g_{\text {best }, i}$ is the best position of all the particles in the swarm. If the initialization condition (5) is satisfied, the method is updated according to (4):

$p_{\text {best } i}=x_{i \mathrm{k}}$

$f\left(x_{i \mathrm{k}}\right)>f\left(p_{\text {best } i}\right)$

where $f$ represents the objective function that should be maximized. The basic PSO algorithm can be explained in five steps:

Step 1: Initialization of the particle position and velocity randomly.

Step 2: Objective function evaluation.

Step 3: $p_{\text {best }}$ and $g_{\text {best }}$ evaluation.

Step 4: Updating of the velocity and position.

Step 5: Repetition of steps 2-4 until the criteria are met.

\section{MPPT ALGORITHM BASED ON NUMERICAL CALCULATION}

In order to find the MPP quickly, and to overcome the problems posed by conventional MPPT algorithms, speed, stability and accuracy, a novel maximum power point tracking controller based on the Lagrangian Interpolation (LI) and a PSO method is proposed. The scheme proposed in this study estimates the voltage value (Vmpp) of the PV module I-V characteristic in the first step, using the constant voltage (CV) method approximation. The $\mathrm{CV}$ method algorithm is the simplest MPPT controller, and usually triggers a quick response. This technique assumes the value of Vmpp at different irradiance points is approximately equal, as shown in Fig.3 [11], [14] and [15].

where Voc represents the open circuit voltage of the PV panel, the ratio between the PV module maximum output voltage, and its open circuit voltage, which are equal to constant $\mathrm{K}$, and assuming that it slightly changed with the solar radiation. A number of authors have suggested good values for $\mathrm{K}$ within the range $0.7-0.92[1]$.

$\frac{V_{m p p}}{V_{o c}}=\mathrm{K}$

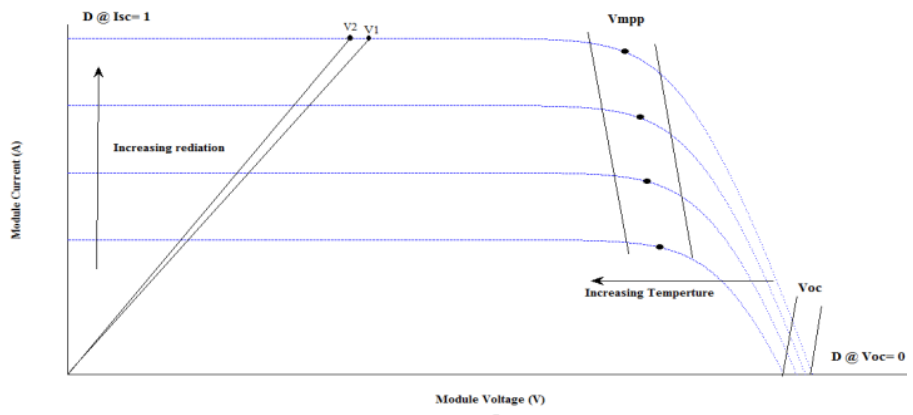

Fig. 3. I-V Characteristic of a photovoltaic cell

The working principle of the algorithm is as follows:

The algorithm begins by obtaining the present value of $V(k)$ and using the previous value, stored at the end of the preceding cycle, $V(k-1)$. Then the value of the duty cycle $d_{m p p}$ at $\left(V_{m p p}\right)$ is estimated, using the Lagrangian interpolation formula, for which four points selected from the (I-V) characteristic are used. Fig.3. represents the PV module (I-V) curve, which is described by the quadratic interpolation function. The interpolation nodes $x_{1}$ and $x_{2}$ represent the voltage values at the two sampling points $\left(V_{1}\right.$ and $\left.V_{2}\right)$, while $x_{0}$ represents the voltage $V_{0}$ of the short circuit current, which is equal to zero, and $x_{3}$ represents the open circuit voltage provided by the PV module data sheet. The function values $y_{1}$ and $y_{2}$ correspond to the voltage values, representing the duty cycle $\left(d_{1}, d_{2}\right)$, the values of the sampling points, and $y_{0}$ and $y_{3}$ represent the duty cycle $\left(\left.d\right|_{I_{S C}}\right.$ and $\left.\left.d\right|_{V_{o c}}\right)$ at the $I_{S c}$ and $V_{o c}$ points, which are equal to 1 and 0 , respectively. Once the values of $V_{0}, V_{1}, V_{2}$ and $V_{o c}$ have been obtained using the aforementioned process, the value of the duty cycle at MPP $d_{m p p}$ at $\left(V_{m p p}\right)$ can be estimated using the Lagrangian interpolation formula. Eq 7 below gives the interpolation formula for $d_{m p p}$ corresponding to $V_{m p p}$ :

$y(x)=\frac{\left(x-x_{1}\right)\left(x-x_{2}\right)\left(x-x_{3}\right)}{\left(x_{0}-x_{1}\right)\left(x_{0}-x_{2}\right)\left(x_{0}-x_{3}\right)} y_{0}+. .+\frac{\left(x-x_{0}\right)\left(x-x_{1}\right)\left(x-x_{2}\right)}{\left(x_{3}-x_{0}\right)\left(x_{3}-x_{1}\right)\left(x_{3}-x_{2}\right)} y_{3}$

where $\mathrm{x}$ is the value of $\mathrm{V}_{\mathrm{mpp}}$. 
Thus, the algorithm for determining the value of $d_{m p p}$ corresponds to $V_{m p p}$. Therefore, the PSO algorithm will trigger the optimisation with an initial value close to the MPP.

\section{A. The Proposed Algorithm}

Unlike conventional techniques, where perturbing and observing power are used to track the PV MPP resulting in long computations time, the proposed algorithm computes the value of initial particles' $d_{M P P}$ (duty cycle at MPP) based on the voltage at maximum power. Therefore, the algorithm can start the optimization process with an initial value that is already close to the MPP. The initial value of particles can be defined as:

$d_{i}{ }^{k}=\left[d_{1}, d_{2}, d_{3}, \ldots \ldots . ., d_{N}\right]$

where $N$ is the number of particles and $k$ is the number of iterations.

To commence the process, the algorithm transmits three duty cycles $d_{1}, d_{2}$, and $d_{3}$ to the Cùk converter; these values are taken as the pbest in the first iteration, and the value closer to the MPP (fitness value) is taken as the gbest value. The duty cycle velocity and position is then updated accordingly. Consequently, when applying the PSO principle, the duty cycle will be perturbed by a small value in the next iteration as a result of comparing the present fitness value with the previous one. This process continues until all particles reach the MPP (a best fitness value) where the velocity is nearly zero.

Since the value of $d_{2}$ is an estimated value computed using (7), and $d_{1}$ and $d_{3}$ are calculated by adding and subtracting a value of $d_{\mathrm{x}}$ from $d_{2}$ to get the upper and lower boundaries, this method leads to a fast dynamic response and accurate tracking. Therefore, a new set of duty cycles can be defined as:

$d_{\text {inew }}=\left[d_{2}-d_{\mathrm{x}}, d_{2}, d_{2}+d_{\mathrm{x}}\right]$

where $d \mathrm{x}$ is chosen to be equal to velocity.

The duty cycles $d 2$ computed using (7) will be very close to the optimum duty cycle. Additionally, because of the earlier PSO exploration, one of $d i(i=1,2,3)$ will always be very close to the best duty cycle. Hence, this allows the PSO to track the new GP rapidly. The two particles ( $d_{1}$ and $\left.d_{3}\right)$ which represent $p_{\text {best, }}$ are too close to $g_{\text {best }}\left(d_{2}\right)$, and so no large change in their velocity is required to come closer to $d 2$. If a sudden change in weather conditions occurs, the duty cycle is then re-initialized, using (9) to set a new duty cycle, which can track a new MPP correctly. The complete flowchart for the proposed method is shown in Fig. 4 and the proposed algorithm uses the following basic principles:

Step 1. Parameter selection: For the proposed MPPT

algorithm, the calculated duty cycle of the converter in (9) is defined as the particle position, and PV module output power is chosen as the fitness value evaluation function.

Step 2. PSO initialization: In a standard initialization, PSO particles are usually randomly initialized. For the proposed MPPT algorithm, the particles are initialized at fixed, equidistant points, positioned around the GP.
Step 3. Fitness evaluation: The fitness evaluation of particle $i$ will be conducted after the digital controller sends the PWM command according to the duty cycle, which also represents the position of particle $i$.

Step 4. Determination of individual and global best fitness: The new calculated individual best fitness (Pbest) and the global best fitness (gbest) of each particle value are compared with previous ones. They are then replaced according to their positions, where necessary.

Step 5. Updating the velocity and position of each particle: The velocity and position of each particle in the swarm is updated according to (2) and (3).

Step 6. Convergence determination: The convergence criterion is checked. If the end criterion is met, the computation will terminate. Otherwise, the iteration is increased by one rerun of Steps 2 through 6.

Step 7. Reinitialization: The convergence criteria in the standard PSO algorithm aim to find the optimal solution or the success of the maximum number of iterations. However, in a PV system, the optimum point is not constant, as it depends on both weather conditions and load impedance. Therefore, the proposed LI-PSO algorithm will reinitialize and search for the new MPP whenever the following conditions are satisfied:

$\left|v_{-}(\mathrm{i}+1)\right|<\Delta \mathrm{v}$

$\left(\mathrm{p} \_\mathrm{i}(\mathrm{k}+1)-\mathrm{p} \_\mathrm{i}(\mathrm{k})\right) /\left(\mathrm{p} \_\mathrm{i}(\mathrm{k})\right)>\Delta \mathrm{p}$ 


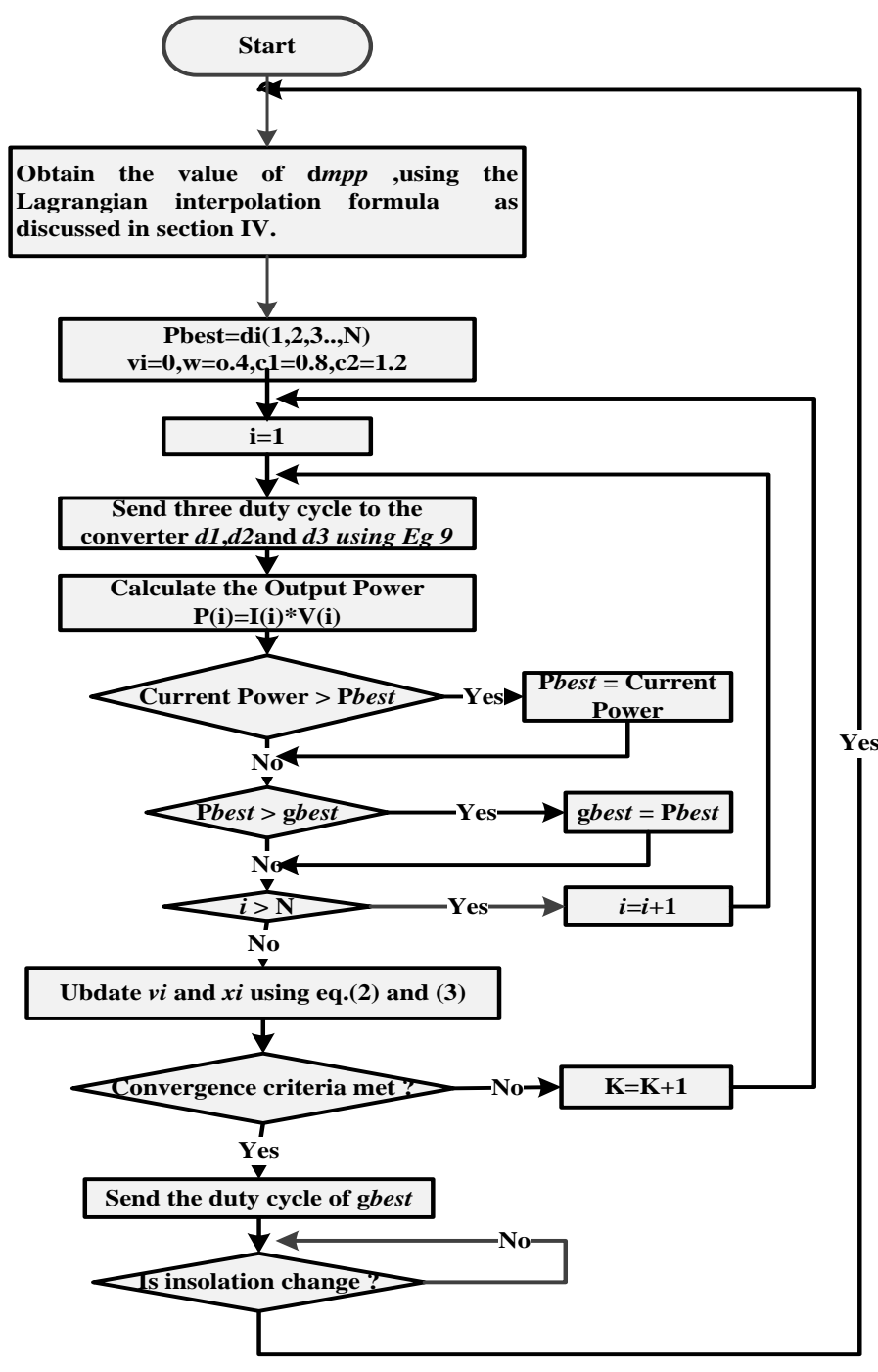

Fig. 4. MPSO algorithm flowchart

where $\mathrm{p}_{-} \mathrm{i}(\mathrm{k}+1)$ is the new $\mathrm{PV}$ power, $\mathrm{p} \_\mathrm{i}(\mathrm{k})$ is the previous PV power at maximum point. Equations (10) and (11) stand for the agent's convergence detection and abrupt alteration of insolation, correspondingly. As already accounted for in [16], there are two matters associated with $\Delta \mathrm{V}$ choice: 1) lesser values lead to better MPPT firmness but a poor tracking reaction, and 2) superior values result in a faster tracking reaction at the cost of greater oscillations. Therefore, a balanced rate must be selected. Nevertheless, when the $\Delta \mathrm{P}$ is great, the subsequent constraint (11) might not be fulfilled due to lesser variations in real power; therefore, the agents' rate of initialization is minor. In accordance with [16] and real-time investigational explorations, the approach to conquering these restrictions and to attaining better tracking performance, is to employ excessive values for $\Delta \mathrm{V}$ and $\Delta \mathrm{P}$, which must be avoided to warrant MPPT stability.

\section{TESTING THE PROPOSED MPPT ALGORITHM}

Figure 5 depicts the main circuit of the hardware-in-loop (HIL) testing platform for the photovoltaic grid-connected inverter. To verify the validity of the proposed MPPT algorithm, the HIL close-loop testing scheme published in [22] is used. The three components of the HIL close-loop testing platform include the RT-LAB simulator. RT-LAB software is used to perform a simulation on the main computer and controller of an inverter connected to a T- type photovoltaic grid. The DSP chip is used with the controller and the digital and analog I/O boards, to join it to the RT-LAB simulator. The PWM pulse is produced by the controller and then travels via the digital input board to the simulator, activating the inverter assembly connected to a 3 level $\mathrm{T}$ type photovoltaic grid [20].

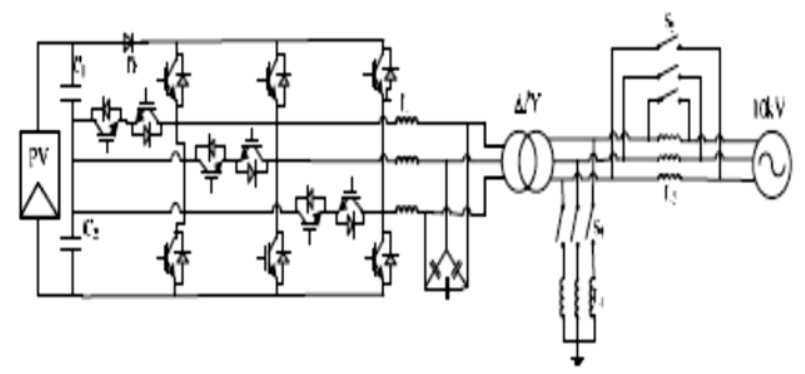

Fig.5 Circuit of hardware-in-loop testing platform

The proposed system was tested in the HIL close-loop, under rapidly changing solar radiation conditions (300 to 1000) Fig.6, and then when the PV array is partially shaded, as shown in Fig.7

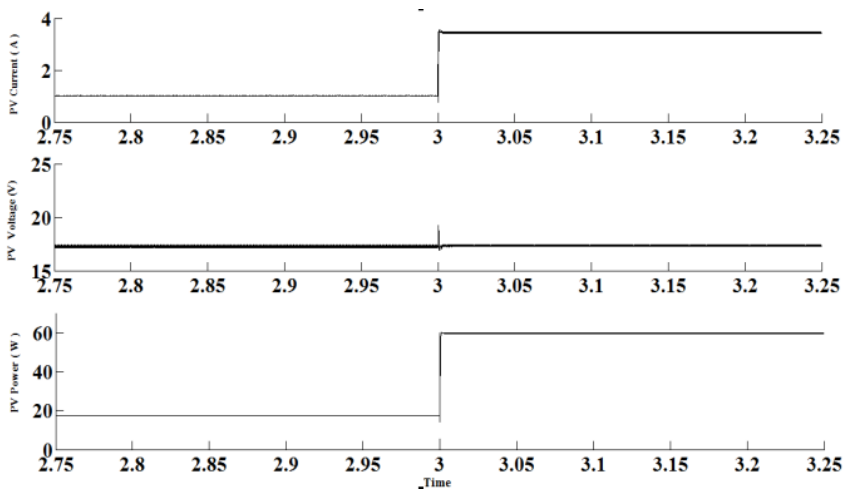

Fig.6. OPAL-RT results of LI-PSO MPPT controller (current, voltage, and power)

The model runs in real-time, with a time-step of $10 \mu$ s for the purpose of control and $135 \mathrm{~ns}$ for the electrical circuit. The PWM pulse was generated at $50 \mathrm{kHz}$. The result was recorded after $250 \mathrm{~ms}$ at 300 and $250 \mathrm{~ms}$ at 1000 . Figure 6 , shows the PV module output current, voltage, and power, under rapidly changing solar radiation conditions (300 to 1000). It can be seen that the proposed algorithm tracked the maximum power level effectively and accurately.
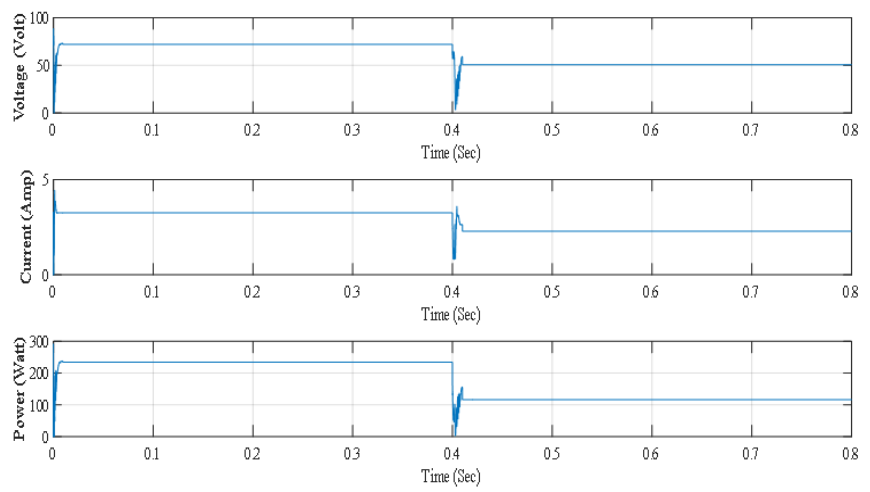
Fig.7. OPAL-RT results of LI-PSO MPPT controller under PSC (current, voltage, and power)

From Fig. 7, it is clear that when partial shading occurs, the LI-PSO algorithm was tracked the true GP P4 (118 W).

\section{DESIGN AND SIMULATION OF MPPT ALGORITHMS}

The proposed system was developed using the Matlab/Simulink and consists of a PV module, and the Ćuk converter, which was chosen as the power interface. The MPPT controller, where the output voltage and current from the PV module are fed into the MPPT algorithm, and subsequently the output of the PWM signal, are used to drive the switch of the Ćuk converter to execute the MPPT from the PV module. There are a number of benefits to this system (1). The entire control mechanism is simplified (2) and so the time taken to perform calculations is decreased (3). Furthermore, there is no requirement to tune PI gains, which enables the system to achieve a fast, dynamic response and reduces its complexity considerably.

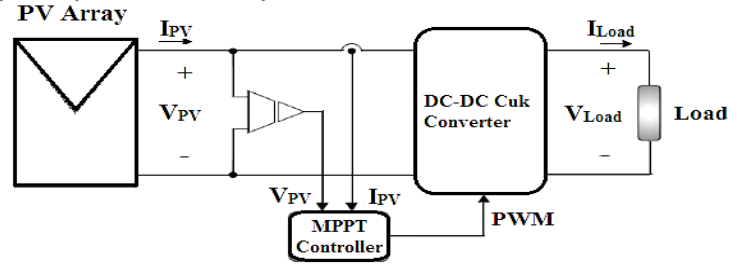

Fig. 8. Simulink model of the MPPT system

To verify the effectiveness of the tracking algorithm and its response time, the proposed system was simulated in Matlab, and the response time for the proposed algorithm was analysed and compared to the P\&O and IncCond methods, and the conventional Particle Swarm Optimization-based MPPT (PSO-MPPT) algorithm. P\&O and IncCond periodically update the duty cycle $d(k)$ applying a fixed step-size of (0.02). The switching frequency of the converter was set to $50 \mathrm{kHz}$. To implement the PSO algorithm and the proposed scheme, the following parameters were used: $C 1=0.8, C 2=1.2, w=$ $0.4, \Delta P=1 \%$, and $\Delta \mathrm{V}=0.4$.

Firstly, the proposed system was simulated with the Matlab model under constant weather conditions, at $\left(1000 \mathrm{~W} / \mathrm{m}^{2}, 25\right.$ $\left.{ }^{\circ} \mathrm{C}\right)$ and $\left(200 \mathrm{~W} / \mathrm{m}^{2}, 25{ }^{\circ} \mathrm{C}\right)$; this was repeated when the PV array was partially shaded, as shown in Fig. 2. Finally, the dynamic performance of the system was studied according to the test conditions addressed in European Standard EN 50530 [22].

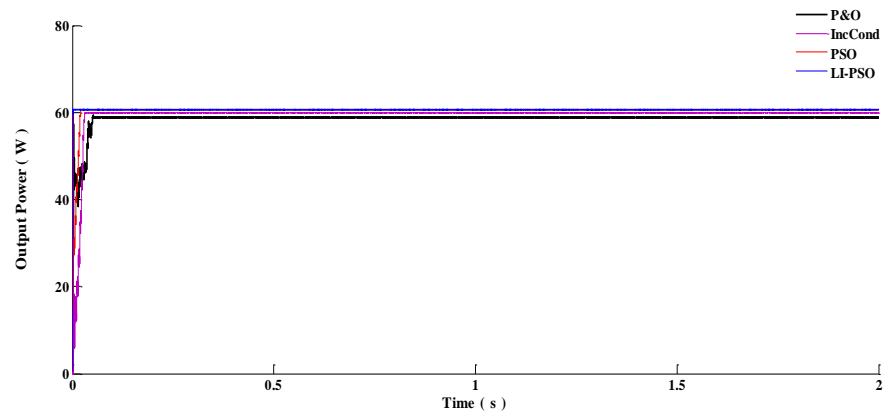

Fig. 9. The PV module output power (W) simulated with Matlab at $\mathrm{G}=1000$ $\mathrm{W} / \mathrm{m}^{2}$ and constant $\mathrm{T}=25^{\circ} \mathrm{C}$
In Fig. 9, it can be observed that the MPP value for the selected PV module is $60.5 \mathrm{~W}$, while it is $60.64 \mathrm{~W}$ with the proposed algorithm. The optimization time for the latter was less than $2 \mathrm{~ms}$, and the convergence speed was also very fast, because the LI-PSO moves the operating point close to the optimal point in a single step. This is unlike conventional techniques where the perturbation and observation of the PV module output power are used to track the MPP. By contrast, the conventional PSO yielded $60.52 \mathrm{~W}$ and required $24 \mathrm{~ms}$ to settle to a new MPP. In that time, the P\&O and IncCond methods yielded values of only $57.76 \mathrm{~W}$ and $59.21 \mathrm{~W}$, respectively. It is clear from the simulation result that the proposed algorithm set the operating point for the MPP ast zero oscillations in a steady state after three iterations.

Figure 10 shows the behaviour of the system under low solar radiation $\left(\mathrm{G}=200 \mathrm{~W} / \mathrm{m}^{2}, \mathrm{~T}=25^{\circ} \mathrm{C}\right)$. It can be seen that the MPP value of the selected PV panel is $11.5 \mathrm{~W}$, while it is $11.64 \mathrm{~W}$ with the LI-PSO algorithm, and the convergence speed is very fast; the conventional PSO was $11.53 \mathrm{~W}$ and its optimization time $35 \mathrm{~ms}$. In that time, the $\mathrm{P} \& \mathrm{O}$ and IncCond methods yielded values of just 10.04 and $10.85 \mathrm{~W}$, respectively. In terms of convergence speed, the proposed method is faster than the conventional PSO algorithm, as the conventional method requires completion of a comprehensive search to set a new MPP.

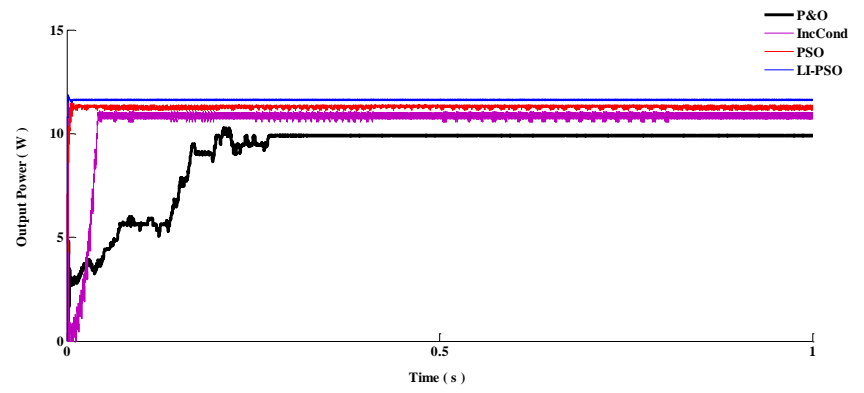

Fig. 10. The PV module output power (W) simulated with Matlab at $\mathrm{G}=200$ $\mathrm{W} / \mathrm{m}^{2}$ and constant $\mathrm{T}=25^{\circ} \mathrm{C}$

Fig. 11, shows the behaviour of the system when the solar radiation levels for the $\mathrm{PV}$ modules were changed from 300 $\mathrm{W} / \mathrm{m}^{2}$ to $500 \mathrm{~W} / \mathrm{m}^{2}$ at a constant temperature of $25^{\circ} \mathrm{C}$. The theoretical value of the MPP, which can be generated from the selected PV module in these cases, is $17.67 \mathrm{~W}$ and $30.35 \mathrm{~W}$, respectively. The changes in solar irradiation occurred at 0.03 $\mathrm{s}$ intervals, and Fig. 12 shows the output power of the system when the radiation was reduced from $800 \mathrm{~W} / \mathrm{m}^{2}$ to $500 \mathrm{~W} / \mathrm{m}^{2}$.

Figs. 11 and 12 show PSO provides an unsuitable response in short periods when there is a gradual change in radiation; this is a common problem affecting the original PSO algorithm.

The dynamic responses of the system output power under varying temperatures of $0^{\circ} \mathrm{C}, 25^{\circ} \mathrm{C}, 70^{\circ} \mathrm{C}$, and $50^{\circ} \mathrm{C}$ are shown in Fig. 13. It is evident that in the case of the LI-PSO MPPT technique, the time taken to set the operating point of the system at its MPP was less than $2 \mathrm{~ms}$ and its tracking efficiencies were higher than $99.94 \%$ in all test conditions, while the conventional PSO was $0.004 \mathrm{~s}$. The proposed technique has provided excellent performance in comparison with other methods; in terms of both dynamic and steady-state responses. 


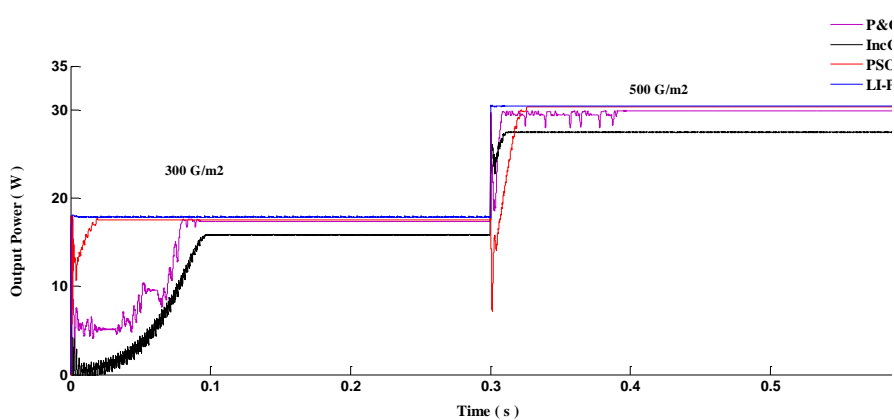

Figure 11: The dynamic response of the output power during rapidly increasing radiation levels.

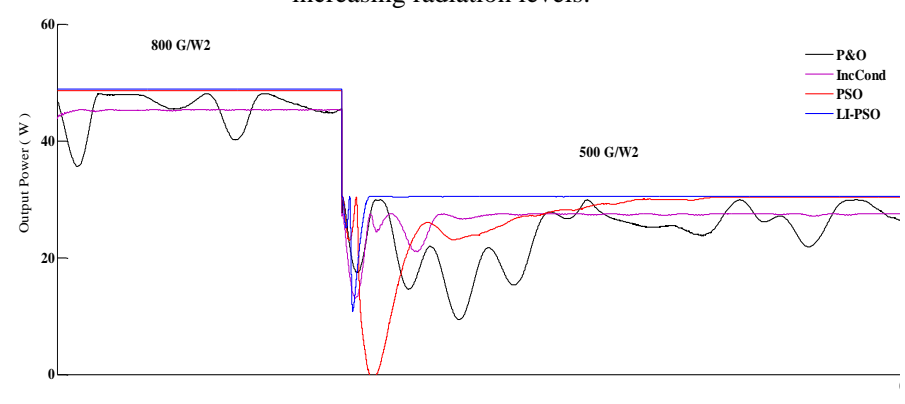

Time ( $\mathrm{s}$

Figure 12: The dynamic response of the output power during rapidly decreasing radiation levels.

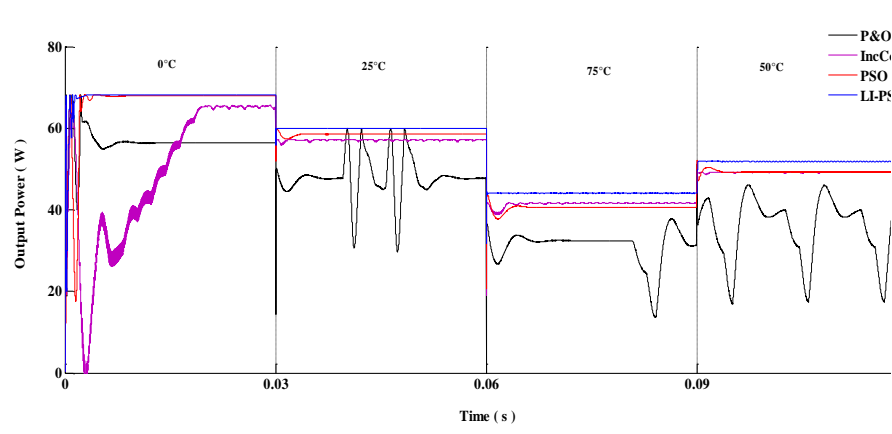

Figure 13: The PV module output power (W) simulated with MATLAB during rapidly changing temperature, $\mathrm{G}=1000 \mathrm{~W} / \mathrm{m}^{2}$.

Table I, summarizes the simulation results for tracked power in (W) between the studied MPPT for different temperatures. It is clear that the power generated when using the proposed algorithm was greater than $98 \%$ under all test conditions.

Table I: Comparison of the studied methods for different temperatures

\begin{tabular}{|c|c|c|c|c|c|}
\hline $\mathbf{T}\left({ }^{\circ} \mathrm{C}\right)$ & P\&O & INC & PSO & LI-PSO & $\begin{array}{c}\text { Theoretical } \\
\text { value of PV }\end{array}$ \\
\hline 0 & 56.32 & 62.95 & 67.88 & 68.22 & 66.45 \\
\hline 25 & 57.76 & 59.21 & 60.52 & 60.64 & 60.5 \\
\hline 50 & 42.65 & 48.85 & 49.52 & 52.84 & 53.08 \\
\hline 75 & 35.59 & 41.68 & 41.04 & 45.62 & 46.18 \\
\hline
\end{tabular}

According to the findings attained, higher efficiency is promoted by either the $\mathrm{P} \& \mathrm{O}$ or the IncCond technique, which both have a fixed step perturbation structure. Nevertheless, in comparison to $\mathrm{P} \& \mathrm{O}$, IncCond produced a slightly better efficiency $(98.3 \%$ vs. $98.5 \%)$. However, at low levels of insolation, both techniques performed poorly, particularly
IncCond, which yielded efficiency levels below $95 \%$ on numerous occasions. Hence, to increase efficiency to $100 \%$, it is necessary to employ adaptive MPPT techniques, which are faster and have minimal fluctuation around the MPP.

The following table provides a comparison of the tracked power in (W), between the theoretical value of the PV module and the MPPT studied for high and low levels of solar radiation. It is clear that the yield energy of the proposed algorithm is above $99.5 \%$ under all test conditions.

Table II: Comparison of the methods studied

\begin{tabular}{|l|l|l|l|l|c|}
\hline $\begin{array}{c}\text { G } \\
\mathrm{W} / \mathrm{m}^{2}\end{array}$ & P\&O & INC & PSO & LI-PSO & $\begin{array}{c}\text { Theoretical } \\
\text { value of PV }\end{array}$ \\
\hline 200 & 10.04 & 10.85 & 11.18 & 11.67 & 11.5 \\
\hline 400 & 14.22 & 19.78 & 24.16 & 24.29 & 24.26 \\
\hline 600 & 33.62 & 33.68 & 36.51 & 36.58 & 36.52 \\
\hline 800 & 42.6 & 43.35 & 48.05 & 48.76 & 48.68 \\
\hline 1000 & 57.76 & 59.21 & 60.22 & 60.64 & 60.5 \\
\hline
\end{tabular}

Fig. 14 illustrates the output power of the techniques studied and proposed under PSC. Initially, the PV was operated at a maximum power of $240 \mathrm{~W}$, and at $t=0.03 \mathrm{~s}$, some of the PV modules in the array were shaded, resulting in four peaks $P_{1}, P_{2}, P_{3}$, and $P_{4}$, where $P_{4}(118 \mathrm{~W})$ is the GP.

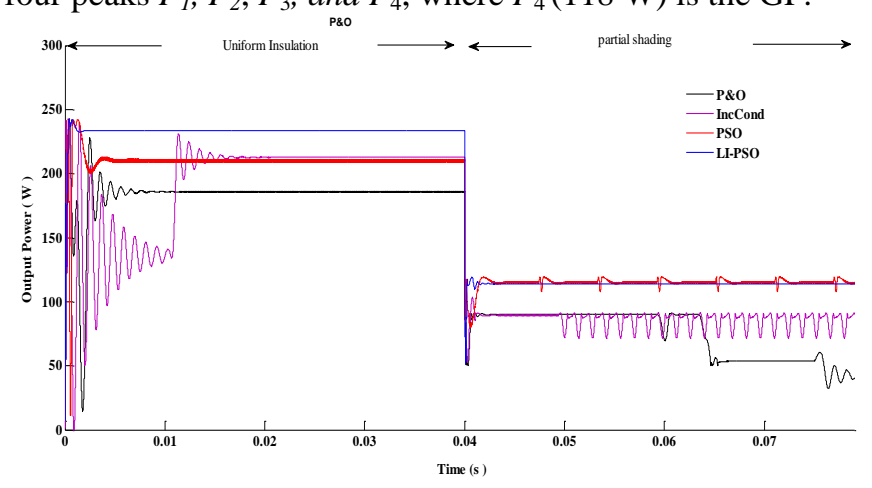

Fig. 14. The PV Module Output Power (w) simulated with the MATLAB Model under PSC.

From Fig. 14, it is clear that when partial shading occurs, the operating point of the $\mathrm{P} \& \mathrm{O}$ was at $\mathrm{P}_{2}(53 \mathrm{~W})$ as the MPP, while both PSO and IncCond are trapped close to the local peak $P_{3}(98 \mathrm{~W})$. However, LI-PSO tracked the true GP $P_{4}$ $(118 \mathrm{~W})$, because the first particle is set to the converged value from the first step, thereby allowing the particles to converge to the GP much faster. Although the conventional PSO-MPPT algorithm is fast and sets the operating point of the system accurately, it is at a disadvantage when searching for MPP with multiple peaks. In this case, however, it was possible when some of the modules were shaded, to track the local MPPs and enable the particles to track the global MPP. In traditional PSO algorithms, the three basic parameters ( $w$, $c 1$ and $c 2$ ) must be tuned in order to accelerate convergence.

As shown in Fig. 15, when the weight (w) was set to a low value, it became apparent that the operating point of the system was at the local $\mathrm{P}_{3}(98 \mathrm{~W})$. This is because a low value for $w$ might cause the particle to suffer from convergence problems, thereby tracking the local optimum instead of the GP. Thus, more iteration is needed to achieve a final solution, because of the distance to the GP. However, as the number of 
iterations increases, the value of $w$ gradually decreases. This, in turn, leads the particles' movement decrease also, leading to a low tracking speed or the aforementioned tracking of a local optimum instead of the GP. Therefore, the value of $w$ in conventional PSO needs to be set to a higher value during the initial search for a good exploration and then this needs to be reduced gradually to allow accurate optimization, while large values for c1, and c2 may cause convergence problems and increase tracking time. Therefore, learning factors and inertia weight in the conventional PSO must be modified when a PSC occurs. However, choosing the appropriate values is challenging, usually requiring experimentation. By contrast, when the PV characteristic changes the proposed algorithm sets the duty cycle close to the optimum in the first step and then PSO locates the GP in the next step, resulting in a shorter tracking time.

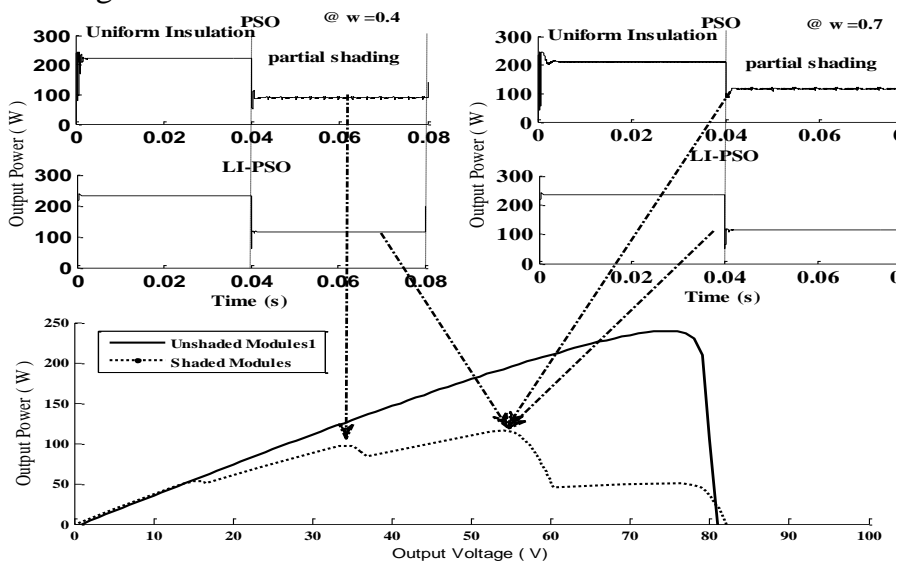

Fig. 15. Tracking performance of PSO and LI-PSO under PSC at $(w=0.4$ and $w=0.7$ ).

Fig. 15 shows the operating point of the system when the $w$ value is changed. It is evident that both the proposed scheme and the conventional PSO were able to operate the system at the exact GP when $w=0.7$, while the conventional PSO was tracked at the local peak instead of the GP when $w=0.4$. This is because the inertia weight was used to control the velocity in the standard PSO, using a constant value of $w$. However, choosing value is an important parameter in PSO, as a large value facilitates a global peak, while a small value facilitates a local optimum.

The test used to calculate the dynamic efficiency of MPPT in different environmental conditions involved using different ramp profiles over a fixed time interval. Fig.16 shows the dynamic performance under two tests, and confirms that the proposed scheme shows the best performance in terms of stability and response time, while the conventional PSO provided better performance compared with $\mathrm{P} \& \mathrm{O}$ and IncCond methods. The $\mathrm{P} \& \mathrm{O}$ method provided the worst Performance, while the IncCond algorithm showed better performance than the $\mathrm{P} \& \mathrm{O}$ algorithm. However, it has a slow response time. It is very sensitive to the perturbation size when low radiation levels occur. Moreover, it is not stable when compared to LI-PSO and conventional PSO algorithms, which duffer from steady state fluctuations, as reported in several works [21, 22]. Both $\mathrm{P} \& \mathrm{O}$ and IncCond MPPT algorithms show weak ability to extract MPP when compared with PSO and LI-PSO, and their tracking efficiency was $97.09 \%$ and $97.97 \%$ respectively.

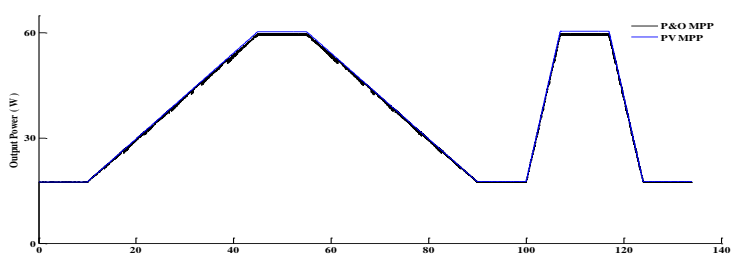

(a)

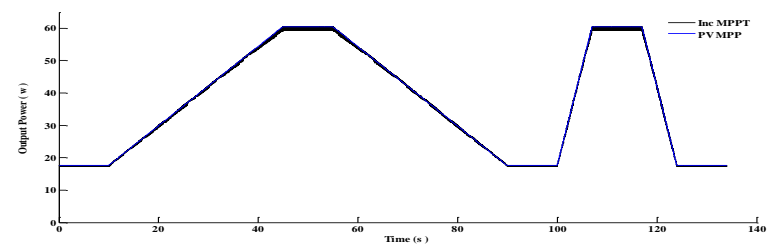

(b)

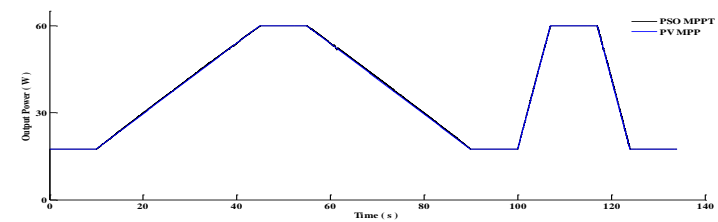

(c)

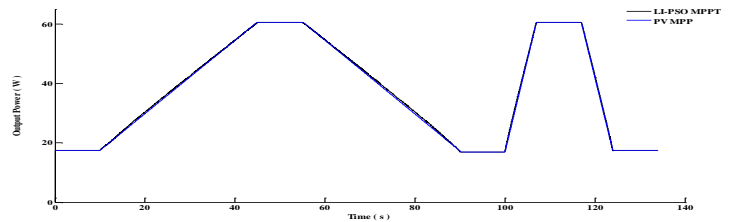

(d)

Fig.16. Dynamic MPPT performances from $30 \%$ to $100 \%$ irradiance. (a) P\&O method. (b) IncCond method. (c) PSO method. (d)LI-PSO method

Table III: Dynamic efficiency

\begin{tabular}{|c|c|c|c|c|c|}
\hline \multirow{2}{*}{\multicolumn{2}{|c|}{ Efficiency $(\%)$}} & \multicolumn{4}{|c|}{ MPPT } \\
\hline & & $\mathrm{P} \& \mathrm{O}$ & IncCond & PSO & LI-PSO \\
\hline \multirow{2}{*}{$\frac{\tilde{a}}{\sqrt{n}}-\frac{\overline{\tilde{n}}}{\xi}$} & 20 & 89.05 & 91.95 & 99.87 & 99.94 \\
\hline & 100 & $\begin{array}{l}97.09 \\
\end{array}$ & 97.97 & 99.92 & 99.97 \\
\hline
\end{tabular}

As indicated in Table III the efficiency of PSO is somewhat lower than with the improved LI-PSO algorithms. Therefore, in this study, it can be confirmed that the best outcomes were acquired using the improved LI-PSO and PSO techniques. Additionally, it is noteworthy that $99.95 \%$ of dynamic efficiency was achieved by utilizing the irradiation slopes. The $\mathrm{P} \& \mathrm{O}$ and IncCond algorithms were found to share close similarity in terms of performance. Hence, a preference for one over the other would be based on simplicity.

From the above figure, it is apparent that the efficiency of the InCond and P\&O Algorithms is low and causes oscillations around the MPP in a steady state, due to the dynamics of the InCond and P\&O Algorithms and the perturbation step size, which is not sufficient to follow the ramp as previously reported [21, 22]. Therefore, to improve their efficiency, adaptive MPPT methods with faster tracking speed should be used. However, they are limited by difficulty finding the closest local maximum power when the PV module is partially shaded, and the only factor in choosing 
them is simplicity. The efficiencies of PSO and the proposed scheme are better when compared with the InCond and P\&O Algorithms, and when tracking the MPP under all ramps. The LI-PSO algorithm results in slightly better performance and has $99.97 \%$ efficiency compare to $99.92 \%$ in PSO.

From the simulation results, it is apparent that the conventional PSO is fast and accurate when searching for single peak values. Nonetheless, when a partial shading condition occurred, the conventional PSO tracking efficiency was low because of the weight $(w)$, which needs to be readjusted correctly. A greater step size in the weighting formula leads to an increase in the particle velocity while a decrease in $w$ causes particle movement to reduce, which enables the controller to locate the operating point for the MPP accurately. Therefore, the parameters of conventional PSO need to be modified when PSC occurs. The difference between the proposed algorithm and the standard PSO is that particles (the duty cycle) are initialised to their optimal value in relation to the MPP. Moreover, it is simple, more precise, and has a faster tracking speed than other methods, and can be implemented using a low-cost digital signal controller (DSC).

\section{CONCLUSION}

In this paper, a mechanism was proposed by which particles can be initialized efficiently around the MPP to avoid both unnecessary and redundant searching and a situation in which the area being actively searched by the swarm becomes too small. The simulation results showed that the proposed LIPSO method results in a faster response rate than other methods. This is because the particles automatically migrate to the best position or move close to it when weather conditions change. As a result, this significantly reduces the time wasted by particle tracking in the wrong area; thereby substantially enhancing the system's tracking speed, while also reducing the steady-state oscillation (practically to zero) once the MPP is located. This is a huge improvement upon the conventional PSO method, in which the new operating point is found too far from the MPP and more iterations are then required to reach the new MPP.

\section{ACKNOWLEDGMENT}

The authors would like to thank Opal-RT Technologies for their technical support and professional help in. providing the research facilities to conduct this research.

\section{References}

[1] B. Subudhi and R. Pradhan, "A Comparative Study on Maximum Power Point Tracking Techniques for Photovoltaic Power Systems", IEEE Trans. Sustain. Energy, vol. 4, no. 1, pp. 89-98, 2013.

[2] M. A. Elgendy, B. Zahawi, and D. J. Atkinson, "Assessment of the incremental conductance maximum power point tracking algorithm," IEEE Trans. Sustain. Energy, vol. 4, no. 1, pp. 108-117, Jan. 2013.

[3] Chikh, Ali, and Ambrish Chandra. "An Optimal Maximum Power Point Tracking Algorithm for PV Systems with Climatic Parameters Estimation." IEEE Trans. Sustain. Energy, vol.6, no. 2 pp.644-652, 2015.

[4] K. S. Tey and S. Mekhilef, "Modified incremental conductance algorithm for photovoltaic system under partial shading conditions and load variation," IEEE Trans. Ind. Electron., vol. 61, no. 10, pp. 5384-5392, Oct. 2014.

[5] Elgendy, Mohammed, Bashar Zahawi, and David J. Atkinson. "Operating Characteristics of the $\mathrm{P} \& \mathrm{O}$ Algorithm at High Perturbation Frequencies for Standalone PV Systems." Energy Conversion, IEEE Transactions,no 30.1, pp 189-198, 2015.

[6] Y.-H. Ji, D.-Y. Jung, J.-G. Kim, J.-H. Kim, T.-W. Lee, and C.-Y. Won, "A real maximum power point tracking method for mismatching compensation in PV array under partially shaded conditions," IEEE Trans. Power Electron., vol. 26, no. 4, pp. 10011009, Apr. 2011.

[7] Sundareswaran, Kinattingal, et al. "Enhanced Energy Output from a PV System under Partial Shaded Conditions through Artificial Bee Colony." IEEE Trans. Sustain. Energy, vol. 6, no. 1, Jan.2015.

[8] Renaudineau, Hugues, Fabrizio Donatantonio, Julien Fontchastagner, Giovanni Petrone, Giovanni Spagnuolo, JeanPhilippe Martin, and Serge Pierfederici. "A PSO-Based Global MPPT Technique for Distributed PV Power Generation." Industrial Electronics, IEEE Transactions on, vol. 62, no. 2, pp 1047-1058 Feb 2015.

[9] K. Ishaque and Z. Salam, "A deterministic particle swarm optimization maximum power point tracker for photovoltaic system under partial shading condition," IEEE Trans. Ind. Electron., vol. 60, no. 8, pp. 3195-3206, Aug. 2013.

[10] Kok Soon Tey and S. Mekhilef, "Modified Incremental Conductance Algorithm for Photovoltaic System Under Partial Shading Conditions and Load Variation", IEEE Trans. Ind. Electron., vol. 61, no. 10, pp. 5384-5392, 2014.

[11] K. Ishaque, Z. Salam, M. Amjad, and S. Mekhilef, "An improved particle swarm optimization (PSO)-based MPPT for PV with reduced steady-state oscillation," IEEE Trans. Power Electron., vol. 27, no. 8, pp. 3627-3638, Aug. 2012.

[12] P. Lei, Y. Li, and J. E. Seem, "Sequential ESC-based global MPPT control for photovoltaic array with variable shading," IEEE Trans. Sustainable Energy, vol. 2, no. 3, pp. 348-358, Jul. 2011.

[13] B. N. Alajmi, K. H. Ahmed, S. J. Finney, and B. W. Williams, "Fuzzylogic-control approach of a modified hill-climbing method for maximum power point in microgrid standalone photovoltaic system," IEEE Trans. Power Electron., vol. 26, no. 4, pp. 1022-1030, Apr. 2011.

[14] K. L. Lian, J. H. Jhang, and I. S. Tian, "A maximum power point tracking method based on perturb-and-observe combined with particle swarm optimization," IEEE J. Photovoltaics, vol. 4, no. 2, pp. 626-633, Mar. 2014.

[15] K. Ishaque, Z. Salam, M. Amjad, and S. Mekhilef, "An improved particle swarm optimization (PSO)-based MPPT for PV with reduced steady-state oscillation," IEEE Trans. Power Electron., vol. 27, no. 8, pp. 3627-3638, Aug. 2012.

[16] M. Miyatake, M. veerachary, F. Toriumi, N. Fujii, and H. Ko, "Maximum power point tracking of multiple photovoltaic arrays: a PSO approach," IEEE Trans. Aerosp. Electron. Syst., vol. 47, no. 1, pp. 367-380, Jan. 2011.

[17] R. B. A. Koad and A. F. Zobaa, "Comparison between the conventional methods and PSO based MPPT algorithm for photovoltaic systems," World Academy of Science, Engineering and Technology, International Science Index 88, International Journal of 
Electrical, Computer, Energetic, Electronic and Communication Engineering, vol. 8, no. 4, pp. 673 - 678.2014.

[18] S. Mirbagheri, M. Aldeen, and S. Saha. "A PSO-based MPPT reinitialised by incremental conductance method for a standalone PV system."Control and Automation (MED), 2015 23th Mediterranean Conference on. IEEE, 2015.

[19] V. Phimmasone, T. Endo, Y. Kondo, and M. Miyatake, "Improvement of the maximum power point tracker for photovoltaic generators with particle swarm optimization technique by adding repulsive force among agents," in Proc. Elect. Mach. Syst., 2009, pp. $1-6$.

[20] Fei, Zheng, Zhang Xiao Lin, Zhang Junjun, and Huang Jingsheng. "Hardware-in-the-loop simulation, modeling and closeloop testing for three-level photovoltaic grid-connected inverter based on RT-LAB." In Power System Technology (POWERCON), 2014 International Conference on, pp. 2794-2799. IEEE, 2014.

[21] Bründlinger R et al. prEN 50530 - The new European standard for performance characterisation of PV inverters. In: 24th Eur Photovoltaic Solar Energy Conf, 2009.

[22] Ishaque, K., Salam, Z., \& Lauss, G, "The performance of perturb and observe and incremental conductance maximum power point tracking method under dynamic weather conditions," Applied Energy,vol 119, pp 228-236.2014.

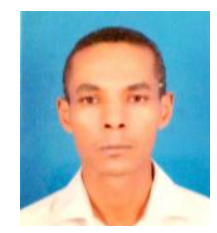

Ramdan B A Koad was born in Ghat, Libya, in 1978. He received the MSc. degrees in electrical Power from the Newcastle University, UK in 2009; he is currently pursuing the Ph.D. degree in renewable energy sources. His main research interests include renewable energy sources and power electronics.

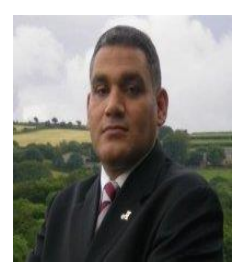

Ahmed Faheem Zobaa received the B.Sc.(Hons.), M.Sc., and Ph.D. degrees in electrical power and machines from Cairo University, Giza, Egypt, in 1992, 1997, and 2002, respectively. From 2007 to 2010, he was a Senior Lecturer in renewable energy with the University of Exeter, Cornwall, U.K. He was also an Instructor from 1992 to 1997, a Teaching Assistant from 1997 to 2002, and an Assistant Professor from 2003 to April 2008 with the Department of Electrical Power and Machines and the Faculty of Engineering, Cairo University, where he has also been an Associate Professor since April 2008. Currently, he is also a Senior Lecturer in power systems with Brunel University, Uxbridge, U.K. His main areas of expertise are lighting applications, power quality, (marine) renewable energy systems, grid integration, smart grids and energy management.

Dr. Zobaa is an Editor-in-Chief for the International Journal of Renewable Energy Technology. He is also an Editorial Board member, Editor, Associate Editor, and Editorial Advisory Board member for many international journals. $\mathrm{He}$ is a registered Chartered Engineer, Chartered Energy Engineer, European Engineer, and International Professional Engineer. He is also a registered member of the Engineering Council U.K., Egypt Syndicate of Engineers, and the Egyptian Society of Engineers. He is a Fellow of the Institution of Engineering and Technology, the Energy Institute of U.K., the Chartered Institution of Building Services Engineers and the Higher Education Academy of U.K. He is a senior member of the Institute of Electrical and Electronics Engineers. He is a member of the International Solar Energy Society, the European Society for Engineering Education, the European Power Electronics and Drives Association, the British Institute of Energy Economics, and the IEEE Standards Association.

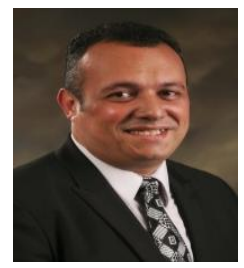

Adel El Shahat (S'08, M'11), Adel El Shahat is currently an Assistant Professor of Electrical Engineering in the Department of Electrical Engineering at Georgia Southern University (GSU), USA. $\mathrm{He}$ is the Founder and Director of the innovative Power Electronics and Nano-Grids Research Lab (iPENG) at GSU. He received his B.Sc. in Electrical Engineering from Zagazig University, Zagazig, Egypt, in 1999. He received his M.Sc. in Electrical Engineering (Power \& Machines) from Zagazig University, Zagazig, Egypt, in 2004. He received his Ph.D. degree (Joint Supervision) from Zagazig University, Zagazig, Egypt, and The Ohio State University (OSU), Columbus, OH, USA, in 2011. His research focuses on various aspects of Smart Grid Systems; Nano \& Micro- Grids; Power Electronics; Electric Machines; Drive Systems; Smart Homes, Distributed Generation; Renewable Energy Systems (Photovoltaic, Wind, ...etc); Power Systems; Energy Storage \& Conservation; Optimization; Neural Networks, Genetic; Power System Stability; Control Systems; Microgenerators Design; Micro-turbine operation; FACTS; Capacitive Deionization; Modeling and Simulation techniques. 